\title{
Elementary Approaches on De Sitter Space
}

\author{
Emre Öztürk* and Yusuf Yaylı
}

\begin{abstract}
In this paper, we characterize the de Sitter space by means of spacelike and timelike curves that fully lies on it. For this purpose, we consider the tangential part of the second derivative of the unit speed curve on the hypersurface, and obtain the vector equations of the geodesics. We find the geodesics as hyperbolas, ellipses, and helices. Moreover, we give an example of null curve with constant curvature in 4 -dimensional Minkowski space and we illustrate the geodesics of $\mathbb{S}_{1}^{1}(r) \times \mathbb{R}$.
\end{abstract}

Keywords: De Sitter space; geodesic; curve with constant curvature

AMS Subject Classification (2010): Primary: 14H45 ; Secondary: 32Q10; $58 E 10$.

${ }^{*}$ Corresponding author

\section{Introduction}

Let we consider $(n+1)$-dimensional Minkowski space $\mathbb{L}^{n+1}=\mathbb{R}_{1}^{n+1}$ endowed with the standart flat metric $d s^{2}=-d x_{1}^{2}+d x_{2}^{2}+\ldots+d x_{n+1}^{2}$, where $x=\left(x_{1}, x_{2}, \ldots, x_{n+1}\right) \in \mathbb{L}^{n+1}$. De Sitter space can be defined as a submanifold of a generalized Minkowski space of one higher dimension. For $r>0$, the $n$-dimensional de Sitter space $\mathbb{S}_{1}^{n}(r)$ is defined as the following hyperquadric of $\mathbb{L}^{n+1}$

$$
\mathbb{S}_{1}^{n}(r)=\left\{x \in \mathbb{L}^{n+1}:\langle x, x\rangle=r^{2}\right\}
$$

The metric on the de Sitter space is the metric induced from the ambient Minkowski metric. The induced metric is nondegenerate and has Lorentzian signature. De Sitter spacetime is the maximally symmetric spacetime of constant positive curvature. It is a solution of the vacuum Einstein equations with a positive cosmological constant. For $n \geq 3$, the de Sitter space $\mathbb{S}_{1}^{n}(r)$ is a simply connected Lorentzian space form with constant sectional curvature $1 / r^{2}$.

In [1], the authors characterized the unit sphere in terms of the curves on hypersurfaces and curvatures. For a closed hypersurface in $\mathbb{R}^{n+1}$, they proved that "If every curve on $M$ has curvature $\geq 1$ and there exits a curve $\gamma$ of lenght $\pi$ with constant curvature 1 on $M$, then $M$ is the unit sphere". Meanwhile, they compared the curvature $\kappa$ of the curve $\gamma$ on a hypersurface and normal curvature at initial point $\gamma(0)$ of the curve. In [6], the authors defined some conditions related with the curves and normal curvature, in the direction of any tangent vector to surface. They stated these conditions as "Every unit speed curve $X(s)$ on hyperbolic space with raidus $r$ satisfies $\left\langle X^{\prime \prime}(s), X^{\prime \prime}(s)\right\rangle \geq-1 / r^{2 \text { " }}$ and "For every unit tangent vector $v$ to $M$, the normal curvature $\kappa_{n}(v)$ in the direction of $v$ satisfies $\left|\kappa_{n}(v)\right| \leq 1 / r^{\prime \prime}$. They used this two conditions for find the geodesics of space and characterize the hyperbolic spaces. In ([2], p.457), condition about the chord property of the curve is given by

$$
\langle X(t)-X(s), T(t)-T(s)\rangle=0
$$

where the $X(s)$ is unit speed curve and $T$ is the unit tangent vector field of the curve. The authors showed that, the curves which satisfy condition above are the curves with constant curvatures. These curves have some interesting properties, for instance, the unit tangent vector field of the curve is given as $T(s)=A X(s)+b$ for any constant skew symmetric matrix $A$ and constant vector $b$. Also the derivatives of $\left|X^{(k)}(s)\right|$ are constant for all $k, 1 \leq k \leq n$,

Received : 27-06-2019, Accepted : 18-08-2019 
in $n$-dimensional Euclidean space. In [9], the unit speed nonnull curves with constant curvature are called as " $C$-curve" in Minkowski space. The authors proved that the norm of the high order derivatives of the $C$-curves are constant and the unit tangent vector field of the curve is $T(s)=\bar{A} X(s)+c$, for suitable constant semi skew symmetric matrix $\bar{A}$, in Minkowski space. In ([8], p.89), O'Neill gave an important result for the geodesics of the product manifolds by the following.

Lemma 1.1. Let $M$ and $N$ be hypersurfaces in Minkowski space. A curve $\gamma(s)=(\alpha(s), \beta(s))$ in $M \times N$ is a geodesic if and only if its projections $\alpha$ in $M$ and $\beta$ in $N$ are both geodesics.

We use this Lemma for state the geodesics of $\mathbb{S}_{1}^{n-1}(r) \times \mathbb{R}$. When we do this, we add the linear term to the equation of geodesics of the de Sitter space.

In [10], null curves that parametrized by the pseudo-arc on $\mathbb{S}_{1}^{n}(r)$ are characterized in terms of Cartan curvatures. The author set up the position vector of the curve with linear combination of Cartan frame. This curve can be represent by

$$
X(s)=b_{1} L+b_{2} N+\sum_{i=1}^{n-1} c_{i} W_{i}
$$

where $\left\{L, N, W_{1}, \ldots, W_{n-1}\right\}$ is Cartan frame and $b_{1}, b_{2}, c_{i}, 1 \leq i \leq n-1$ are coefficients. By initial condion of $\langle X(s), X(s)\rangle=r^{2}$, permanently differentiating the confront equations gives the Cartan curvatures $\left\{k_{1}, k_{2}, \ldots, k_{m}\right\}$ depends on the coefficients in (1.1).

In this study, we mean with "curve fully lies on de Sitter space" that the curves lie on boundary of de Sitter space. That is, we consider the de Sitter space as timelike surface. In the light of [6], we characterize the de Sitter space $\mathbb{S}_{1}^{n-1}(r)$, by considering null and nonnull curves that fully lies on it. For this purpose, we consider the tangential part of the second derivative of the unit speed curve on the hypersurface and the normal curvature in the direction of any tangent vector to surface. Consequently, we obtain the vector equations of planar and helical geodesics of the de Sitter space and $\mathbb{S}_{1}^{n-1}(r) \times \mathbb{R}$. Throughout the study, we try to answer some questions like following, ?"

"What is the characteristic property of the curves and geodesics that fully lies on de Sitter space and $\mathbb{S}_{1}^{n-1}(r) \times \mathbb{R}$

We prove that the planar and helical nonnull geodesics that fully lies on de Sitter space and $\mathbb{S}_{1}^{n-1}(r) \times \mathbb{R}$, are the $C$-curves, that is curve with constant curvature. Moreover, we show that all null geodesics on de Sitter space are nothing but the lines.

Throughout this article, all curves regular, and all hypersurfaces smooth and connected, unless otherwise mentioned.

\section{Characterization by Timelike and Spacelike Curves}

Let $\bar{\nabla}$ and $\nabla$ be the Levi-Civita connections on $\mathbb{L}^{n+1}$ and the hypersurface $M$, respectively. Consider that, $Z$ is the unit normal vector field on $M$ and $S$ is the shape operator of the hypersurface. It follows from Gauss equation that

$$
\bar{\nabla}_{V} W=\nabla_{V} W+\varepsilon_{M}\langle S(V), W\rangle Z
$$

where $V, W \in \chi(M)$ and $\varepsilon_{M}=\langle Z, Z\rangle$ (see [7], p.77). $\nabla_{V} W$ and $\varepsilon_{M}\langle S(V), W\rangle Z$ are called the tangential and the normal part of $\bar{\nabla}_{V} W$, respectively.

Let $X(s)$ be a unit speed nonnull curve on the timelike hypersurface $M$ and $X^{\prime}(s)$ be a tangent vector to $M$ at $X(s)$. Since $M$ is timelike, $\varepsilon_{M}=1$ (i.e. $Z$ is spacelike). If $V=W=X^{\prime}(s)$ in equation (2.1) then we have

$$
X^{\prime \prime}(s)=k_{g}(s)+\left\langle S\left(X^{\prime}(s)\right), X^{\prime}(s)\right\rangle Z(s)
$$

where $k_{g}(s)$ is the tangential part of $X^{\prime \prime}(s)$. Hence, equation (2.2) becomes

$$
X^{\prime \prime}(s)=k_{g}(s)+\left\langle X^{\prime \prime}(s), Z(s)\right\rangle Z(s)
$$

It follows from equation (2.3) that every geodesic curve $X(s)$ on timelike hypersurfaces satisfy

$$
X^{\prime \prime}(s)=\left\langle X^{\prime \prime}(s), Z(s)\right\rangle Z(s)
$$

Moreover, any unit tangent vector $v$ to $M$, the normal curvature in the direction of $v$ is given by $\kappa_{n}(v)=\langle S(v), v\rangle$. Hence, it follows from $v=X^{\prime}(s)$ and (2.2) that

$$
X^{\prime \prime}(s)=k_{g}(s)+\kappa_{n}\left(X^{\prime}(s)\right) Z(s)
$$


Lemma 2.1. Suppose that $k_{g}(s)$ is spacelike or timelike vector for all $s$. Then, every unit speed curve on timelike hypersurface $M$ satisfy $\left\langle X^{\prime \prime}(0), X^{\prime \prime}(0)\right\rangle=\kappa_{n}(v)^{2}, X^{\prime}(0)=v$ if and only if $X(s)$ is a geodesic curve on $M$.

Proof. Let $X(s)$ be a geodesic curve on $M$. Consider that $p \in M$ and unit tangent vector $v$ to $M$ such that $X(0)=p$ and $X^{\prime}(0)=v$. It follows from $k_{g}(s)=0$ and (2.5) that

$$
X^{\prime \prime}(0)=\kappa_{n}(v) Z(s)
$$

and this implies the left side.

If $\left\langle X^{\prime \prime}(0), X^{\prime \prime}(0)\right\rangle=\kappa_{n}(v)^{2}$, it follows from equation (2.5) and hypothesis, that $k_{g}(s)=0$. Hence, $X(s)$ is a geodesic.

Lemma 2.2. Let $X(s)$ be a unit speed curve on timelike hypersurface $M$ and the tangential part of $X^{\prime \prime}(s)$ satisfy $\left\langle k_{g}(s), k_{g}(s)\right\rangle \leq$ 0 , then, the following conditions are equivalent:

$(C)$ Every unit speed curve $X(s)$ on $M$ satisfies $\left\langle X^{\prime \prime}(s), X^{\prime \prime}(s)\right\rangle \leq \frac{1}{r^{2}}$.

$(N)$ For every unit tangent vector $v$ to $M$, the normal curvature $\kappa_{n}(v)$ in the direction of $v$ satisfies $\left|\kappa_{n}(v)\right| \leq \frac{1}{r}$.

Proof. For any unit tangent vector $v$ to $M$ at $p$, we consider the geodesic $X$ with $X(0)=p$ and $X^{\prime}(0)=v$. It follows from Lemma 2.1 that

$$
\left\langle X^{\prime \prime}(0), X^{\prime \prime}(0)\right\rangle=\kappa_{n}(v)^{2} \leq \frac{1}{r^{2}}
$$

Thus, $(C)$ implies $(N)$.

Conversely, $\left\langle X^{\prime \prime}(s), X^{\prime \prime}(s)\right\rangle=\left\langle k_{g}(s), k_{g}(s)\right\rangle+\kappa_{n}\left(X^{\prime}(s)\right)^{2} \leq \kappa_{n}\left(X^{\prime}(s)\right)^{2} \leq \frac{1}{r^{2}}$ where the inequality follows from $\left\langle k_{g}(s), k_{g}(s)\right\rangle \leq 0$.

Theorem 2.1. Suppose that the unit speed curve $X(s)$ on timelike hypersurface $M$ satisfies condition $(C)$ with $\left\langle k_{g}(s), k_{g}(s)\right\rangle \leq$ 0. If $\left\langle X^{\prime \prime}(s), X^{\prime \prime}(s)\right\rangle=\frac{1}{r^{2}}$, then, it is a periodic parametrization of an ellipse given by

$$
X^{1}(s)=\xi_{1}^{1} \sin \frac{s}{r}+\xi_{2}^{1} \cos \frac{s}{r}+\xi_{3}^{1}
$$

or hyperbola given by

$$
X^{2}(s)=\xi_{1}^{2} \sinh \frac{s}{r}+\xi_{2}^{2} \cosh \frac{s}{r}+\xi_{3}^{2}
$$

for some vectors $\xi_{i}^{j} \in \mathbb{L}^{n+1}, 1 \leq i \leq 3,1 \leq j \leq 2$.

Proof. Suppose that a unit speed curve $X(s)$ on $M$ satisfies the condition $(C)$. It follows from Lemma 2.2 that

$$
\left\langle X^{\prime \prime}(s), X^{\prime \prime}(s)\right\rangle=\left|k_{g}(s)\right|^{2}+\kappa_{n}\left(X^{\prime}(s)\right)^{2} \leq\left|k_{g}(s)\right|^{2}+\frac{1}{r^{2}}
$$

Together with (2.8), the hypothesis $\left\langle X^{\prime \prime}(s), X^{\prime \prime}(s)\right\rangle=\frac{1}{r^{2}}$ implies that $X(s)$ is a geodesic with $\kappa_{n}\left(X^{\prime}(s)\right)=\frac{1}{r}$. Thus, from equation (2.5) we have

$$
X^{\prime \prime}(s)=\frac{1}{r} Z(s)
$$

Since $\kappa_{n}\left(X^{\prime}(s)\right)=\frac{1}{r}$ is a maximum value of normal curvatures at $X(s), X^{\prime}(s)$ is a principal direction of $M$. Besides, the equation $\kappa_{n}\left(X^{\prime}(s)\right)=\left\langle S\left(X^{\prime}(s)\right), X^{\prime}(s)\right\rangle=\frac{1}{r}$ implies that

$$
S\left(X^{\prime}(s)\right)=\frac{1}{r} X^{\prime}(s)
$$

for spacelike $X(s)$ curves. Moreover, we have $S\left(X^{\prime}(s)\right)=-Z^{\prime}(s)$. Thus, it follows from (2.9) and (2.10) that

$$
X^{\prime \prime \prime}(s)=-\frac{1}{r^{2}} X^{\prime}(s)
$$

Solutions of (2.11) gives the $X^{1}(s)$.

On the other hand, for timelike $X(s)$ curves, $S\left(X^{\prime}(s)\right)=-\frac{1}{r} X^{\prime}(s)$. Likewise we get $X^{2}(s)$. 
Theorem 2.2. Let $M$ be a timelike hypersurface in Minkowski space. Then, up to isometries of $\mathbb{L}^{n+1}, M$ is de Sitter space with radius $r$ if and only if there exits a point $p \in M$ such that for every unit speed geodesic $X(s)$ of $M$ through the point $p$, $\left\langle X^{\prime \prime}(s), X^{\prime \prime}(s)\right\rangle=\frac{1}{r^{2}}$ holds and $X(s)$ satisfies the $(C)$ with $\left\langle k_{g}(s), k_{g}(s)\right\rangle \leq 0$.

Proof. Suppose that the unit speed curve $X(s)$ satisfies the condition $(C)$ with $\left\langle k_{g}(s), k_{g}(s)\right\rangle \leq 0$ and $\left\langle X^{\prime \prime}(s), X^{\prime \prime}(s)\right\rangle=$ $1 / r^{2}$ holds for all unit speed geodesic $X(s)$ of $M$ through the point $p$. Then, by isometries of $\mathbb{L}^{n+1}$, we may assume that $p=(0, \ldots, 0, r)$ and the unit normal $Z(p)$ at $p$ is given by $Z(p)=(0, \ldots, 0,1)$. For any arbitrary point $q \in M$, the completeness of $M$ implies that there exits a unit speed geodesic $X(s)$ of $M$ connecting $p$ and $q$ with $X(0)=p$. Together with the hypothesis, Theorem 2.1 shows that for some vectors $\xi_{i}^{j} \in \mathbb{L}^{n+1}, 1 \leq i \leq 3,1 \leq j \leq 2$, the geodesic $X(s)$ is given by $X^{1}(s)$ or $X^{2}(s)$. Hence, we get

$$
\left(X^{1}\right)^{\prime}(s)=\frac{\xi_{1}^{1}}{r} \cos \frac{s}{r}-\frac{\xi_{2}^{1}}{r} \sin \frac{s}{r}
$$

and

$$
\left(X^{2}\right)^{\prime}(s)=\frac{\xi_{1}^{2}}{r} \cosh \frac{s}{r}+\frac{\xi_{2}^{2}}{r} \sinh \frac{s}{r}
$$

Since the geodesic $X(s)$ is of unit speed

$$
\left\langle\xi_{1}^{1}, \xi_{2}^{1}\right\rangle=0, \quad\left\langle\xi_{1}^{1}, \xi_{1}^{1}\right\rangle=\left\langle\xi_{2}^{1}, \xi_{2}^{1}\right\rangle=r^{2}
$$

and

$$
\left\langle\xi_{1}^{2}, \xi_{2}^{2}\right\rangle=0, \quad-\left\langle\xi_{1}^{2}, \xi_{1}^{2}\right\rangle=\left\langle\xi_{2}^{2}, \xi_{2}^{2}\right\rangle=r^{2}
$$

It follows from $X^{\prime \prime}(s)=\frac{1}{r}\left\langle X(s), X^{\prime \prime}(s)\right\rangle Z(s)$ and the assumption $Z(p)=(0, \ldots, 0,1)$ that $\xi_{1}^{1}=(0, \ldots, 0, r), \xi_{2}^{1}=r u$ in (2.6), and $\xi_{1}^{2}=(r, 0, \ldots, 0), \xi_{2}^{2}=r \bar{u}$ in (2.7) for some unit vectors, $u=\left(u_{1}, u_{2}, \ldots, u_{n-1}, 0\right)$ and $\bar{u}=\left(0, u_{1}, u_{2}, \ldots, u_{n-1}\right)$ respectively. Together with the initial condition $X(0)=p$, this shows that $X(s)$ is given by

$$
X^{1}(s)=(0, \ldots, 0, r) \sin \frac{s}{r}+r u \cos \frac{s}{r}
$$

or

$$
X^{2}(s)=(r, 0, \ldots, 0) \sinh \frac{s}{r}+r \bar{u} \cosh \frac{s}{r}
$$

We have $\left\langle X^{i}(s), X^{i}(s)\right\rangle=r^{2}$, for $i=1,2$ which means $X(s)$ lie on $\mathbb{S}_{1}^{n-1}(r)$.

Conversely, all curves on $M$ satisfies $\langle X(s), X(s)\rangle=r^{2}$. Since $Z(s)=X(s) / r$, by straightforward calculations, we get the right side.

Corollary 2.1. The geodesics of fully lies on $\mathbb{S}_{1}^{n-1}(r) \times \mathbb{R}$ are the helices given by

$$
X^{3}(s)=\xi_{1}^{3} \sin \frac{s}{r}+\xi_{2}^{3} \cos \frac{s}{r}+\xi_{3}^{3} s+\xi_{4}^{3}
$$

and

$$
X^{4}(s)=\xi_{1}^{4} \sinh \frac{s}{r}+\xi_{2}^{4} \cosh \frac{s}{r}+\xi_{3}^{4} s+\xi_{4}^{4}
$$

for some vectors $\xi_{i}^{3}, \xi_{i}^{4} \in \mathbb{L}^{n+1}, 1 \leq i \leq 4$.

Proof. The geodesics of $\mathbb{S}_{1}^{n-1}(r)$ are given by equations (2.6) and (2.7). It is obvious that the lines are geodesic in $\mathbb{R}$. It follows from Lemma 1.1, the geodesic curves that fully lies on $\mathbb{S}_{1}^{n-1}(r) \times \mathbb{R}$ are given by (2.18) and (2.19).

Corollary 2.2. The geodesics on de Sitter space and $\mathbb{S}_{1}^{n-1}(r) \times \mathbb{R}$ are the $C$-curves.

Proof. The geodesics of $\mathbb{S}_{1}^{n-1}(r) \times \mathbb{R}$ are given by $X^{i}(s), 1 \leq i \leq 4$. Let we consider the curve,

$$
X^{4}(s)=\xi_{1}^{4} \sinh \frac{s}{r}+\xi_{2}^{4} \cosh \frac{s}{r}+\xi_{3}^{4} s+\xi_{4}^{4}
$$


The unit tangent vector field of $X^{4}(s)$ is given by

$$
T(s)=\frac{\xi_{1}^{4}}{r} \cosh \frac{s}{r}+\frac{\xi_{2}^{4}}{r} \sinh \frac{s}{r}+\xi_{3}^{4}
$$

It follows from (2.20) and (2.21) that

$$
\left\langle X^{4}(t)-X^{4}(s), T(t)-T(s)\right\rangle=0
$$

Hence, $X^{4}(s)$ is a $C$-curve as part of the definition. In other cases, the proof is similar.

\section{Null Curves and Some Examples}

In this section, we follow the previous section to find all null curves on de Sitter space. We characterize these curves in terms of the second derivative of the curve. Moreover, we give an example, a null curve on $\mathbb{S}_{1}^{3}(1)$, and we give some illustrations of geodesics. We note that, in the literature, there are various approachs for null curves such that the subject depends the choice of the Frenet frame of the curve (see [3], [4], and [5]).

Let us consider the curve $X(s)$ as a null curve on $\mathbb{S}_{1}^{n-1}(r)$. Since $X(s)$ is null curve $\left\langle X^{\prime}(s), X^{\prime}(s)\right\rangle=0$. Differentiating $\langle X(s), X(s)\rangle=r^{2}$ twice yields $\left\langle X^{\prime \prime}(s), X(s)\right\rangle=0$. From (2.3) and $Z(s)=X(s) / r$, we get $X^{\prime \prime}(s)=k_{g}(s)$, for all $s$. In the light of ([3], Chapter 3), we have

$$
\left\langle k_{g}(s), k_{g}(s)\right\rangle \geq 0
$$

for all null curves on $\mathbb{S}_{1}^{n-1}(r)$.

We say that a null curve $X(s)$ in $\mathbb{L}^{n+1}$ is parametrized by the pseudo-arc if $\left\langle X^{\prime \prime}(s), X^{\prime \prime}(s)\right\rangle=1$. Based on (3.1), we should examine such two conditions $\left\langle k_{g}(s), k_{g}(s)\right\rangle=0$ and $\left\langle k_{g}(s), k_{g}(s)\right\rangle>0$. Assume that $\left\langle k_{g}(s), k_{g}(s)\right\rangle \neq 0$, then $\left\langle k_{g}(s), k_{g}(s)\right\rangle>0$, and

$$
u(s)=\int_{s_{0}}^{s}\left\langle X^{\prime \prime}(s), X^{\prime \prime}(s)\right\rangle^{1 / 4} d s
$$

becomes the pseudo-arc parameter (see, [10]). Let $X(s)$ be a null curve parametrized by the pseudo-arc on $\mathbb{S}_{1}^{2}$. Then it can be shown easily that $X(s)$ is a line, that is, all null curves on $\mathbb{S}_{1}^{2}$ are just the line. Also these curves are geodesics on hyperboloid in 3-dimensional Minkowski space (see, [5], p.59-60). In higher dimensions, null curves on $\mathbb{S}_{1}^{n-1}$ are more rich from three dimensional Minkowski space (see Example 3.1). For characterization of all null curves, in terms of Cartan curvatures, on $\mathbb{S}_{1}^{n-1}, n \geq 4$, see [10]. On the other hand, if we suppose that $X(s)$ is a geodesic curve or say equivalently $k_{g}(s)=X^{\prime \prime}(s)=0$ then the hypothesis conclude that $X(s)$ is a line on $\mathbb{S}_{1}^{n-1}(r)$. Consequently, all null geodesic curves on $\mathbb{S}_{1}^{n-1}(r)$ are nothing but the lines.

Now, let us give some examples, curves in the de Sitter space.

Example 3.1. Let we give the curve $X(s)$ such that

$$
X(s)=\left(\frac{1}{\sqrt{2}} \sinh s, \frac{1}{\sqrt{2}} \cosh s, \frac{1}{\sqrt{2}} \sin s, \frac{1}{\sqrt{2}} \cos s\right)
$$

Obviously $\langle X(s), X(s)\rangle=1$, that is $X(s)$ lies on $\mathbb{S}_{1}^{3}(1)$. If we check the causality of $X$ we get $\left\langle X^{\prime}(s), X^{\prime}(s)\right\rangle=0$. Hence, $X(s)$ is a null curve in three dimensional de Sitter space with radius 1 . Moreover, this curve satisfy

$$
\langle X(t)-X(s), T(t)-T(s)\rangle=0
$$

in Minkowski space. This leads to extend the definition of $C$-curves, that is, we can consider that $X(s)$ is a null $C$-curve in three dimensional de Sitter space. According to Euclidean metric we get

$$
\langle X(t)-X(s), T(t)-T(s)\rangle=(\cosh s-\cosh t)(\sinh s-\sinh t)
$$

that means $X$ is not $C$-curve in Euclidean space.

Example 3.2. Let us give the intersection of $\mathbb{S}_{1}^{2}$ and some planes in $\mathbb{L}^{3}$. For instance, the intersection of $\mathbb{S}_{1}^{2}(1)$ and a plane with a slope less than 1 is an ellipse (Figure 1a), and a plane with a slope equal to 1 containing the origin is parallel lines (Figure 1b). 


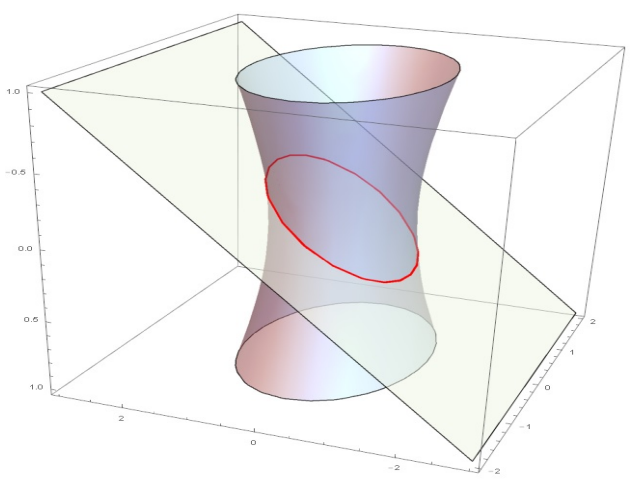

(a) A plane with slope less than 1

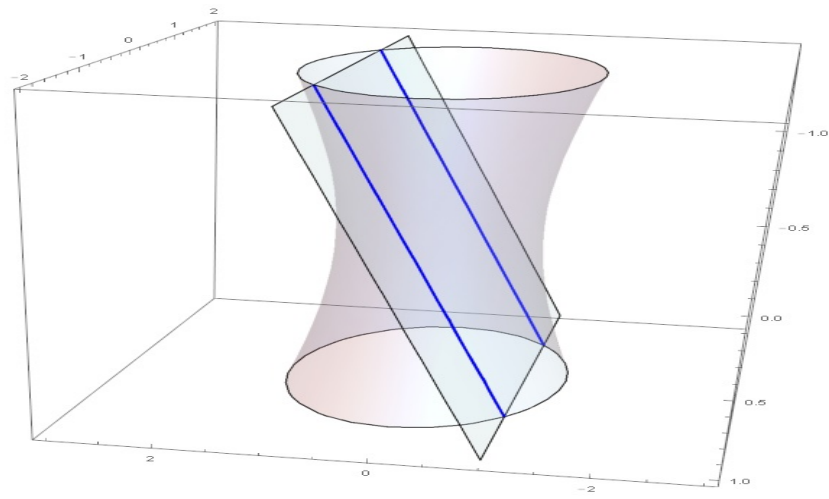

(b) A plane with slope 1

Figure 1. Intersection of hyperboloid with planes

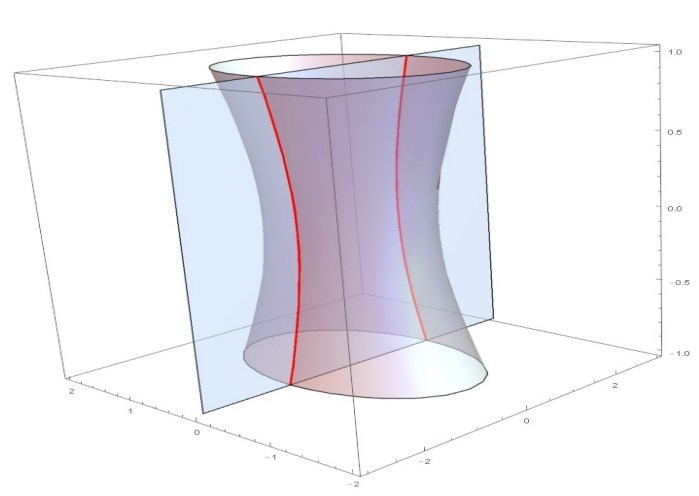

(a) Hyperbola

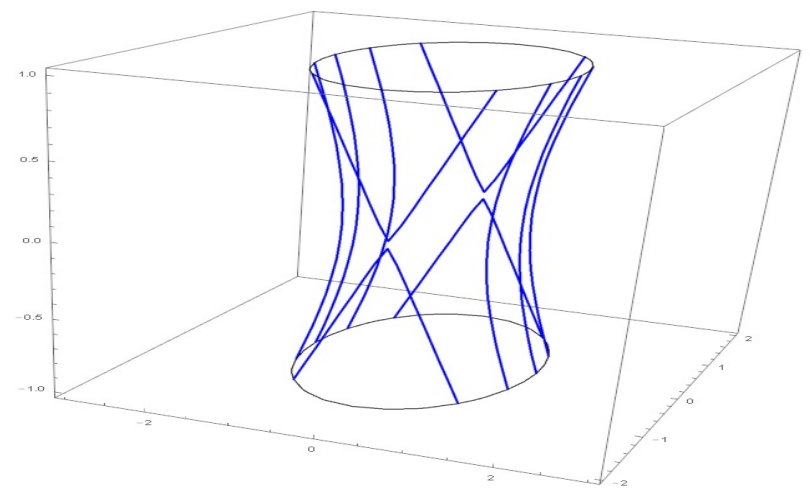

(b) Pair of lines

Figure 2. Cross section and contour plot of hyperboloid

The intersection of $\mathbb{S}_{1}^{2}(1)$ and a non-tangential plane with a slope greater than 1 is hyperbola (Figure 2a), and a tangential plane is a pair of lines (Figure $2 b$, by contour plot). Moreover, the intersection of $\mathbb{S}_{1}^{2}(1)$ and a plane with a slope equal 1 not containing the origin is a parabola and a plane orthogonal to axis of hyperboloid is circle.

Example 3.3. We give sample of spacelike and timelike geodesics of $\mathbb{S}_{1}^{1}(2) \times \mathbb{R}$ by

$$
X_{1}(s)=(s / 2,2 \sin s, 2 \cos s)
$$

and

$$
X_{2}(s)=(2 \sinh s, 2 \cosh s, s)
$$

respectively (see Figure 3a and Figure 3b).

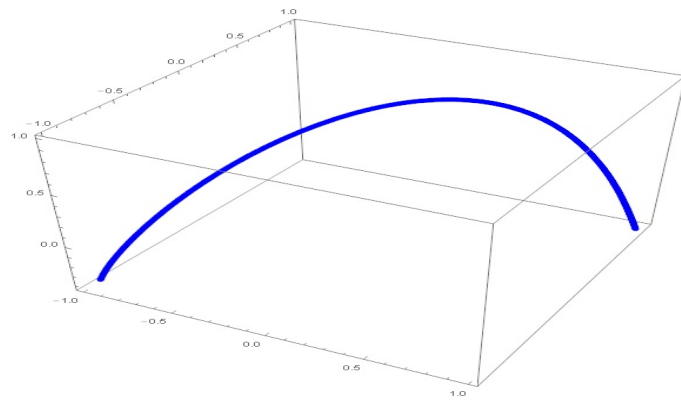

(a) Spacelike helix

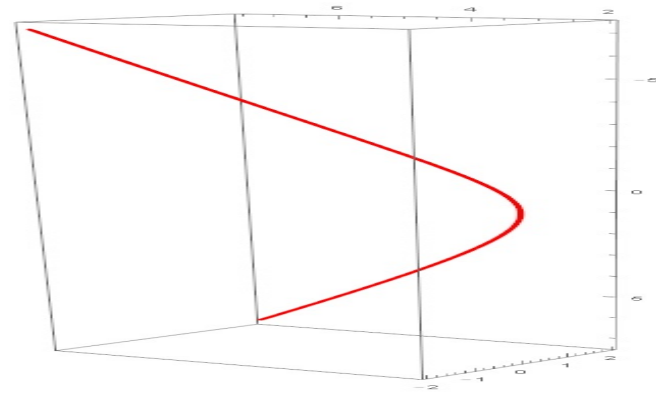

(b) Timelike helix

Figure 3. Spacelike and timelike geodesics of $\mathbb{S}_{1}^{1}(2) \times \mathbb{R}$ 
Our last picture is about null geodesics of $\mathbb{S}_{1}^{1}(1 / 2) \times \mathbb{R}$ and $\mathbb{S}_{1}^{1}(2 / 3) \times \mathbb{R}$ such that these curves are given as

$$
X_{3}(s)=\left(\frac{1}{2} \sinh 2 s, \frac{1}{2} \cosh 2 s, s\right)
$$

and

$$
X_{4}(s)=\left(\frac{2}{3} \sinh 3 s, \frac{2}{3} \cosh 3 s, 2 s\right)
$$

by Figure 4.
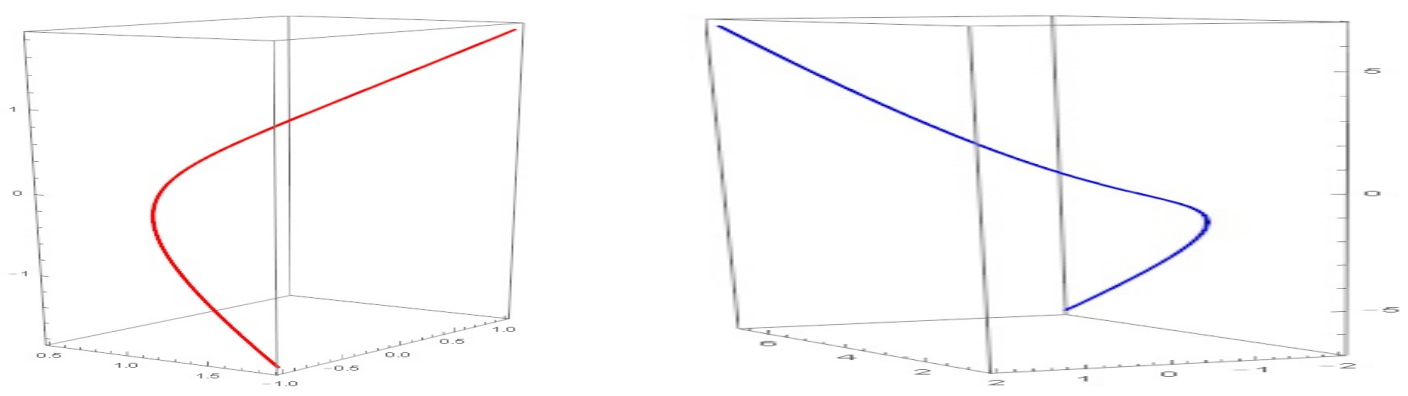

Figure 4. Null helices of product space

\section{References}

[1] Baek, J., Kim, D.S. and Kim, Y. H., A characterization of the unit sphere, Amer. Math.Monthly, 110(2003), no. 9, 830-833.

[2] Chen, B.Y., Kim, D.S. and Kim,Y.H., New Characterizations of W-Curves, Publ. Math. Debrecen, 69 (2006), no. 4, 457-472.

[3] Duggal, K. L. and Bejancu, A.Lightlike Submanifolds of Semi-Riemannian Manifolds and Applications, Kluwer 1996.

[4] Ferrandez, A., Gimenez, A.and Lucas, P., Null helices in Lorentzian space forms, Internat. J.Modern Phys. A, 16(2001), no. 30, 4845-4863.

[5] Inoguchi, J. and Lee, S., Null curves in Minkowski 3-space, Int. Electron. J. Geom., 1(2008), no. 2, 40-83

[6] Kim, D.S., Kim, Y.H. and Lee, J.W. A Characterization of Hyperbolic Spaces, Bull. Korean Math. Soc., 55(2018), no. 4, 1103-1107

[7] Lopez, R., Differential Geometry of Curves and Surfaces in Lorentz-Minkowski space, Int.Electron. J. Geom., 7(2014), no.1, 44-107.

[8] O’Neill, B., Semi-Riemannian Geometry with Applications to Relativity, Academic Press. Inc., 1983.

[9] Öztürk, E. and Yaylı, Y., W-Curves In Lorentz-Minkowski Space, Mathematical Sciences and Applications E-Notes, 5(2017), no.2, 76-88.

[10] Sakaki, M., Notes on null curves in Minkowski space, Turkish Journal Math, 34(2010), 417-424, doi:10.3906/mat0812-14 


\section{Affiliations}

EMRE ÖZTÜRK

AdDress: Turkish Court of Accounts, 06520, Ankara-Turkey.

E-MAIL: emreozturk1471@gmail.com

ORCID ID: 0000-0001-6638-3233

YUSUF YAYLI

AdDRESS: Ankara University, Dept. of Mathematics, 06100, Ankara-Turkey.

E-MAIL: yayli@science.ankara.edu.tr

ORCID ID: 0000-0003-4398-3855 